\title{
Chapter 19 \\ Combining Techniques for Remotely Assessing Pelagic Nekton: Getting the Whole Picture
}

\author{
James Churnside, Richard Brodeur, John Horne, Patrick Adam, \\ Kelly Benoit-Bird, Douglas C. Reese, Amanda Kaltenberg, \\ and Evelyn Brown
}

\begin{abstract}
A variety of observational techniques either have been developed or are under development for fisheries research. These techniques have greatly increased the quantity and quality of information that can be obtained from a research survey and it is anticipated that this trend will continue. Traditional ship-based surveys will be supplemented by data collected from fixed moorings, autonomous underwater vehicles, aircraft, and satellites. Each of these platforms is limited in the spatial and temporal scales that can be sampled. By combining data from multiple platforms and sensors, we will be able to obtain a more complete picture of the components of a particular ecosystem over a greater range of scales. This is particularly true for pelagic nekton, which can move independent of fluid motion. In many cases, the observational difficulties created by this mobility can be mitigated by the use of aircraft, which can cover large areas with optical instruments such as imagers and Light Detection and Ranging (lidar).
\end{abstract}

Keywords Fisheries surveys $\bullet$ lidar $\bullet$ pelagic nekton

\author{
J. Churnside \\ NOAA Earth System Research Laboratory, CS3, 325 Broadway, Boulder, CO 80305, USA \\ e-mail: james.h.churnside@noaa.gov \\ R. Brodeur, D. C. Reese \\ Northwest Fisheries Science Center, National Marine Fisheries Service, \\ Hatfield Marine Science Center, Newport, OR 97365, USA \\ e-mails: Rick.Brodeur@noaa.gov, dreese@ lifetime.oregonstate.edu \\ J. Horne, P. Adam \\ University of Washington, School of Fisheries, Box 355020, Seattle, WA 98195, USA \\ e-mails: jhorne@u.washington.edu, patrick1@u.washington.edu
}

K. B.-Bird, A. Kaltenberg

College of Oceanic and Atmospheric Sciences, Oregon State University, 104 COAS Admin

Bldg, Corvallis, OR 97331-5503, USA

e-mails: kbenoit@coas.oregonstate.edu, akalnteb@coas.oregonstate.edu

E. Brown

Flying Fish Ltd., P.O. Box 169, Husum, WA 98623, USA

e-mail: husumbandb@earthlink.net

R.J. Beamish and B.J. Rothschild (eds.), The Future of Fisheries Science in North America, 345 Fish \& Fisheries Series, () Springer Science + Business Media B.V. 2009 


\subsection{Introduction}

Fisheries science has always utilized, and often initiated, technological and analytical developments in sampling and data processing. Sampling technologies for pelagic species have traditionally focused on specialized hardware, with each device designed to sample a single or a suite of conspecifics. As the development of individual instruments matured, additional sensors or samplers were often added to a common platform. Data collection among instruments has remained largely autonomous, with data integration occurring after collection.

Continued increases in computing power and data storage have expanded the potential for the acquisition of large data sets. Expanded acquisition often includes higher sample resolution, extended ranges of sampling, or the addition of data streams from the same or colocated instruments. A significant challenge when collecting data from multiple instruments is to synchronize and integrate sampling over space and time. In the recent past, advances in sampling technologies have shifted from an emphasis on increased data acquisition to the analysis and interpretation of the data collected.

At the present time, much of what we know about the distribution of pelagic fishes and plankton is based on data from trawl or ship-mounted acoustic surveys along transects (Gunderson 1993). Both survey methods are conducted from vessels that are relatively slow-moving and are vulnerable to aliasing, depending on the direction of a survey relative to movements by species of interest. Data from trawl surveys are constrained by unknown species-specific gear selectivity and the movement and aggregation patterns of the target animals. Acoustic data are constrained by the lack of ability to differentiate among species and to detect targets at boundaries such as the water surface and the bottom (Fréon and Misund 1999). Near-surface areas inhabited by many pelagic species are particularly difficult to sample with conventional downward-looking echosounders due to the depth of the transducers, the dead zone right below the transducer face, and potential vessel avoidance by fish dwelling near the surface.

While it is clear from this volume that there is a wide variety of tools available for fisheries research, no single instrument and/or platform is capable of providing a complete picture of a marine ecosystem. One problem is that there is only a limited range of spatial and temporal scales that can be accessed from each of the available platforms; the other is that there is only a limited amount of information that can be obtained by each of the available instruments. One solution to the constraints of single sensors and platforms is to integrate and combine information obtained by various techniques to obtain the "whole picture." The epipelagic ecosystem presents particular challenges because of the mobility of its constituents. It also has the unique property that many of its constituents can be observed using airborne optical techniques such as light detection and ranging (lidar), which provides access to a range of spatial and temporal scales not possible from other platforms. 


\subsection{Techniques}

For the purposes of this chapter, we will define a technique as a piece of equipment and the platform used to deploy it. By definition, a hull-mounted echosounder installed on a ship is a technique that is different from the same echosounder deployed on a fixed mooring. The first example provides spatial information about the distribution of fish, while the second provides temporal information. Similarly, an echosounder installed on a ship is a technique different from a trawl on the same ship. The information obtained from each of the two sampling gears is very different and even though the sampling equipment is on a common platform, the spatial and temporal scales sampled by the two gears are also different. Clearly, some techniques are more appropriate than others to describe the spatial and temporal dynamics of the pelagic ecosystem, but there is no single technique that is capable of providing the complete picture at all spatial and temporal scales. In this chapter, we consider combinations of techniques that can be used to sample the pelagic habitat. Various techniques have been combined in the past, but we see the future of fisheries research as involving increased numbers and synchrony of combinations of techniques.

Nets and other sampling gear will always be a critical component of fisheries research. Recent developments in net technology will increase the range and spatial resolution of net sampling (Godø 2007, this volume). However, these are limited to deployment from surface ships, with a corresponding limit to the area that can be covered.

Vessel-mounted acoustics are used regularly in resource assessment and ecosystem description. Transducers from echosounders or sounders may be permanently attached to the hull of a vessel or mounted on a platform that is towed by the ship. Hull-mounted acoustic systems are typically maintained by large government institutions that conduct assessment and research on commercially important fish and invertebrates. Because all acoustic surveys include a direct sampling component, pelagic and demersal fish trawls, plankton nets, and water sampling instruments such as conductivity-temperature-depth (CTD) recorders are commonly found on acoustic research vessels. Acoustic technologies such as echosounders or multibeam sonars may also be mounted on small platforms, commonly called towed bodies. These instrument packages may be dedicated to acoustic sampling or may contain other biological and physical samplers (e.g., Wiebe et al. 2002). A towed package has the advantages of sampling waters close to the surface and can be used on any vessel. Hull-mounted systems are typically able to sample in rougher weather, are installed on vessels that include all instruments needed to conduct a full survey, and contain the infrastructure that integrates data collection and storage. Recent developments in acoustics are providing better target discrimination and calibration than ever before, and further improvements are predicted (Foote 2007, this volume; Holliday 2007, this volume).

Optical techniques are particularly well suited for aircraft deployment. The advantage of aircraft is that large areas of the ocean can be covered quickly at a small fraction of the cost per kilometer of a surface ship. Infrared radiometers provide sea-surface temperature (SST), which is an important physical characteristic of the 
epipelagic environment. Visible radiometers provide estimates of the primary productivity of the near-surface layer. Visible imaging systems detect schools of fish near the surface. Lidar detects fish and plankton deeper in the water column.

Aircrafts have been used by the fishing industry for many years to efficiently locate fish (e.g., herring, sardine, and menhaden) and to direct fishing effort by purse seiners. The objective of the fishers is to locate dense concentrations of fish close to a processing plant, a sampling strategy that starkly contrasts from that of a fisheries abundance or research survey. Because schools of epipelagic fish are visible from the air (Fig. 19.1), species identification is generally possible using school morphology, color, and characteristic flashing patterns of individual fish.

The application of lidar to fisheries research is a relatively new technique (Brown et al. 2002; Carrera et al. 2006; Churnside et al. 2001, 2003; Churnside and Thorne 2005; Churnside and Wilson 2004; Lo et al. 2000; Tenningen et al. 2006). The National Oceanic and Atmospheric Administration (NOAA) fish lidar installation is shown in Fig. 19.2. A schematic diagram of the lidar (Fig. 19.3) shows the major components. A short $(12 \mathrm{~ns})$ pulse of green $(532 \mathrm{~nm})$ light is generated by the laser. The light from the laser is pointed about $15^{\circ}$ from nadir by a pair of steering mirrors, which provides alignment with the receiver telescope. The beam is also expanded to be eye-safe for humans and marine mammals (Zorn et al. 2000) at the water surface. Light scattered from the ocean is polarization-filtered and collected by a telescope. Polarization orthogonal to the transmitted polarization is typically used because it provides the highest contrast between fish and small particles in the water (Vasilkov et al. 2001). The telescope is focused using an

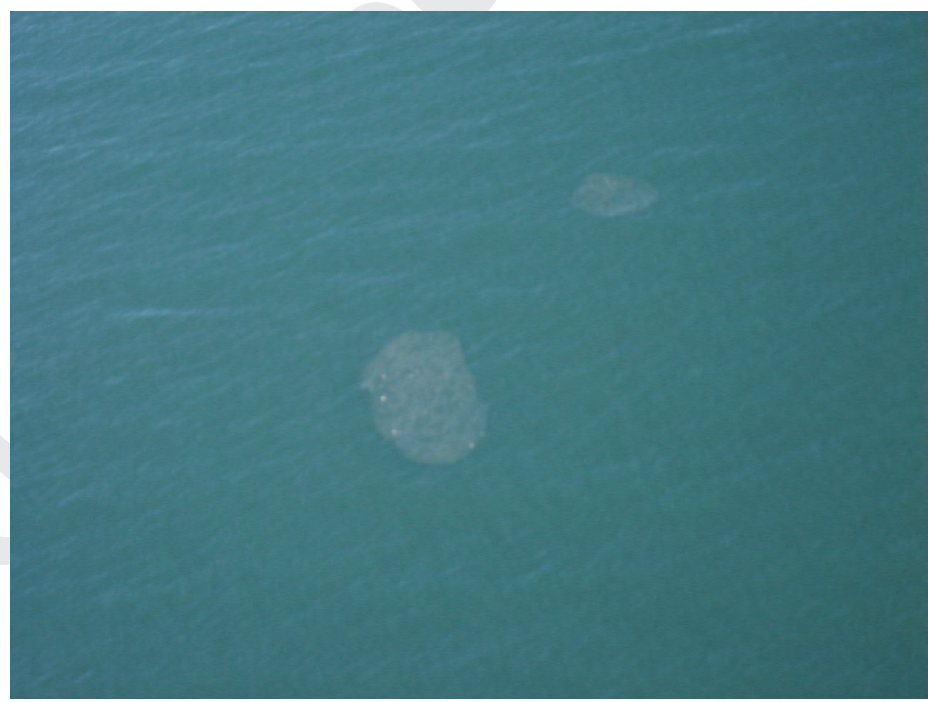

Fig. 19.1 Aerial photo of two schools of menhaden in the Atlantic Ocean about $10 \mathrm{~km}$ east of Ocean City, New Jersey. The larger school is about $15 \mathrm{~m}$ in the long direction (Photo courtesy of Brian Lubinsky, US Fish and Wildlife Service) 


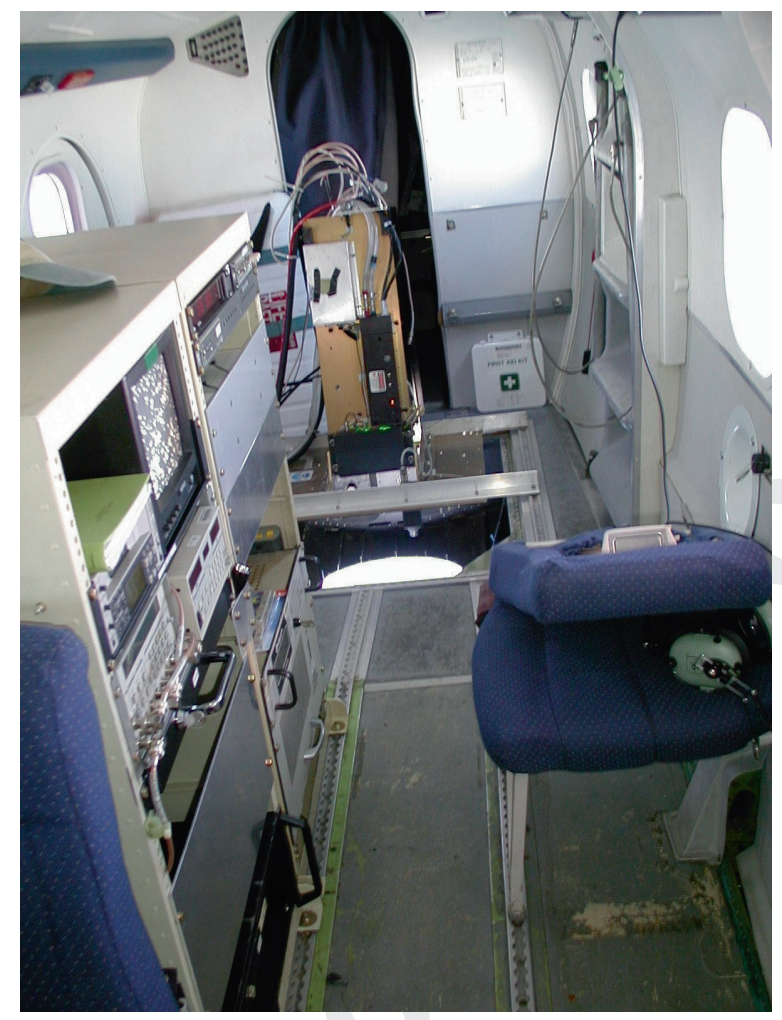

Fig. 19.2 Installation of fish lidar in the NOAA Twin Otter aircraft

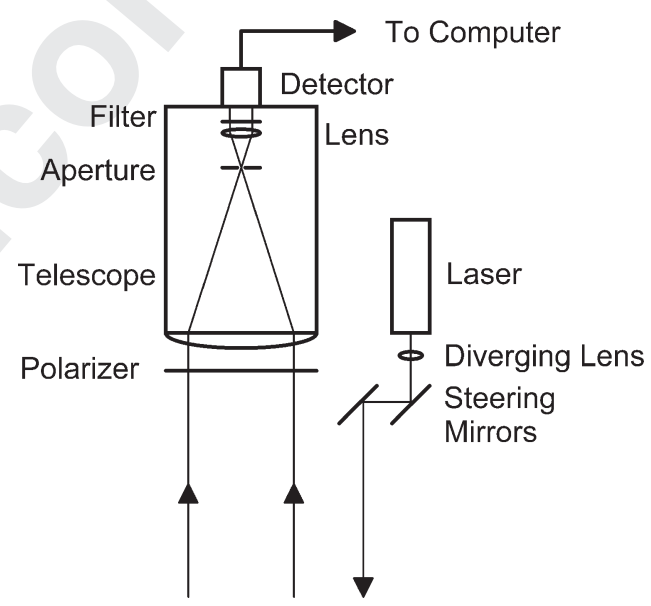

Fig. 19.3 Schematic diagram of the NOAA fish lidar 
interference filter, which reduces the amount of background light, onto a photomultiplier tube, which converts the light into an electronic signal. This signal is digitized at a rate of $1 \mathrm{GHz}$, producing a profile of the scattering in the water with a depth resolution of $11 \mathrm{~cm}$. A second receiver channel collects the scattered light that is co-polarized with the transmitter to provide more information about small-particle scattering.

Data from lidar are similar to echosounder data, in that they provide a depth profile of returned or backscattered energy. It is important to note that the propagation and scattering physics of light and sound differ. The main consequence of this is that lidar can only penetrate to about $50 \mathrm{~m}$ depth in the clearest waters, and even less in typical coastal waters. Depending on the carrier frequency, scientific echosounders can sample the water column over several hundred meters. Figure 19.4 (a-d) presents several examples of scattering profiles from the lidar.

(a)

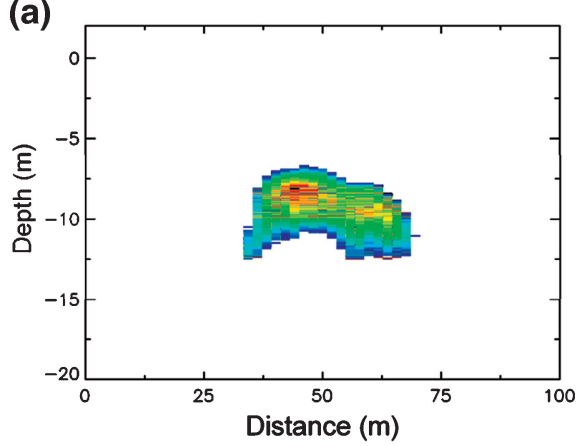

(c)

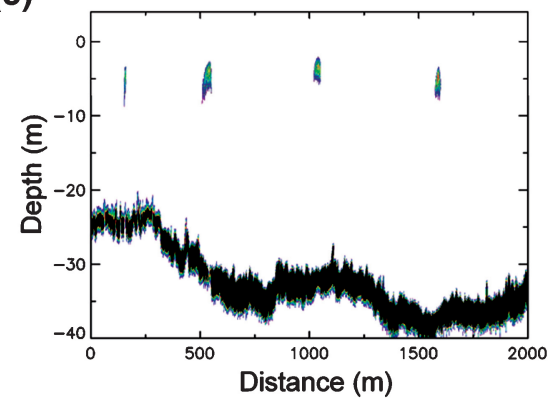

(b)

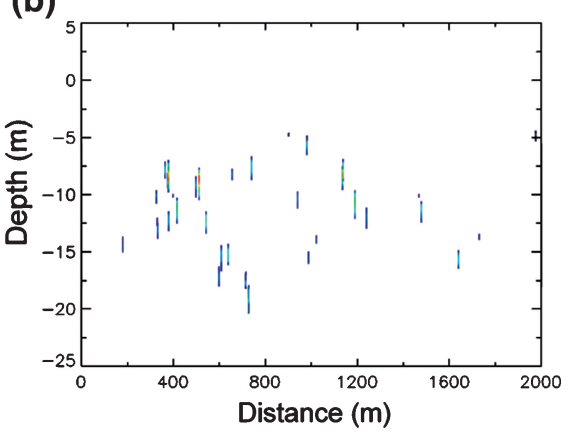

(d)

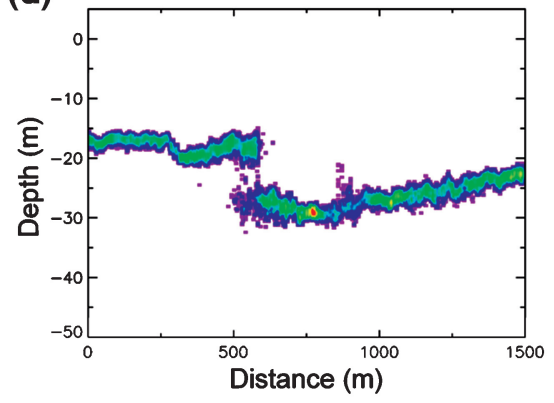

Fig. 19.4 Examples of fish school backscatter profiles from lidar, with relative color scale at the bottom. (a) School of sardines off the Oregon coast; (b) aggregation of salmon sharks in the Gulf of Alaska; (c) four schools of herring over the bottom in the southeastern Bering Sea (note the bottom return is black where it is off the color scale); (d) thin zooplankton layer off the Oregon coast 
Data from optical techniques are also acquired from satellites. Sea-surface temperature and primary productivity maps are currently available. Lidar from satellites may be possible in the future, although the ability to resolve small fish schools with this technique is unlikely.

\subsection{Combinations of Techniques}

Combining sampling equipment on the same platform is the easiest way to combine and integrate techniques. The spatial and temporal range of the data will match while sample resolution will depend on instrument aperture and sample frequency. Matching sample data using a common resolution is easier when samples are synchronized on the same platform. Another relatively straightforward integration of data types is the combination of ship and satellite data. Despite the huge difference in platform speed $\left(10 \mathrm{~km} \mathrm{~s}^{-1}\right.$ for a satellite in low-earth orbit compared with $5 \mathrm{~m} \mathrm{~s}^{-1}$ for a surface ship), the spatial and temporal resolutions of the two data sources are compatible. Aircraft provide access to large areas in short time frames, which results in a more synoptic data stream than ships can provide, but the ability to combine aircraft and ship data is more difficult.

A traditional combination of sampling techniques places acoustics and fishing gear on the same ship. A systematic sampling of preplanned transects is occasionally interrupted to sample dense concentrations of all observed acoustic targets. The systematic acoustic survey provides information on fish distribution, while samples from trawls are used to collect data on species composition, length distribution, and physiological or reproductive condition of fish.

Another combination of sampling techniques uses video and acoustic equipment mounted on trawl nets. These gears are used to monitor fish behavior around and in the net. These techniques are discussed elsewhere in this volume (Godø 2007, this volume).

The combination of vessel-mounted and moored acoustics can be used to collect spatially and temporally independent density data. Vessel-mounted acoustics generate spatially indexed data that may be confounded by the passage of time while sampling, but is typically used to map density distributions in an area, estimate abundance, and to size fish or invertebrate populations of interest. Moored acoustics are fixed in space but spatial coverage is restricted by the transducer beam angle and the depth of the water column. Moored acoustics are used to investigate vertical movement patterns over daily cycles or the timing and flux of migrations (Onsrud et al. 2005). An array of moorings can be placed to capture horizontal movement patterns if a priori knowledge of migration direction is available. Moored acoustic arrays, typically deployed on a smaller scale (e.g., $3 \mathrm{~km}$ in length with $0.5 \mathrm{~km}$ spacing) compared to ship-based acoustic surveys, which may cover hundreds or thousands of nautical miles, are planned to expand in both scope and coverage (e.g., see http://www.oceantrackingnetwork.org/index.html). 
A limited number of surveys have combined vessel-based and moored acoustics. These studies have typically been limited to a single, downward-looking echosounder, such as the Bergen Acoustic Buoy system (Skaret et al. 2006) as the moored component. In this configuration, the acoustic buoy records a time series of nekton density, but the directional component of nekton horizontal flux remains unknown. To detect the horizontal flux of mesopelagic nekton, Benoit-Bird and Au (2004) used a linear array of upward-looking moored echosounders. Even more detailed information about seasonal fish movements and predator-prey interactions has been obtained by a combination of acoustic sensors in a Norwegian fjord (Godø 2007, this volume).

To investigate the four-dimensional flux of nekton, we have combined vesselmounted and moored acoustics in a small-scale grid (1 nautical square mile) survey. One upward-looking $200 \mathrm{kHz}$ acoustic system was moored at each corner of the grid enabling the vertical, latitudinal, and longitudinal movement of nekton to be resolved over 7 days at a resolution of $15 \mathrm{~min}$. To complement data recorded by the moored acoustics, the spatial distribution of nekton within the survey grid was surveyed along transects using 38,120, and $200 \mathrm{kHz}$ echosounders. Coincident measurements were made at each of the four corners, providing observations of spatially and temporally indexed densities. The vessel completed sets of eight transects (four north-south, four east-west) to record the fine-scale distribution of nekton within the survey grid and to estimate the horizontal flux of nekton through the survey area.

Radiometers in low-earth orbit provide maps of sea-surface temperature and primary productivity of near-surface waters. These radiometers do not cover the entire earth during each orbit, but build a composite image over a period of approximately 1 week. With consideration of data losses due to cloud cover, a monthly composite may be required to complete an image. This time period is very similar to the timescale of a vessel-based acoustic survey. In both cases, the assumption is made that there are no significant changes in the density distributions of the species or ecosystem components during the survey time period. An example of the combination of satellite data with an acoustic/trawl ship survey (Fig. 19.5) shows how the backscattered acoustic energy is concentrated in the upwelling zone near the coast. The trawl results are used to relate the backscattered energy to the length and density distributions of sardines.

Understanding pelagic ecosystems is difficult due to the sheer volume of these environments, making it problematic and costly to sample. As mentioned above, satellites provide coverage over large spatial scales; however, complete coverage is often disrupted by cloud cover. One way to overcome this dilemma is to combine the satellite data with data obtained from aircraft. Aircraft are capable of covering large areas over short periods of time, thus making them ideal for environments that are typically difficult or costly to sample. By combining satellite and aircraft data, it is possible to obtain data on animal distributions along with important habitat characteristics such as SST and chlorophyll concentrations, over large spatial scales at high temporal resolutions. In addition, for areas which are not sampled by satellites (e.g., due to cloud cover or areas close to shore) information can be obtained by 


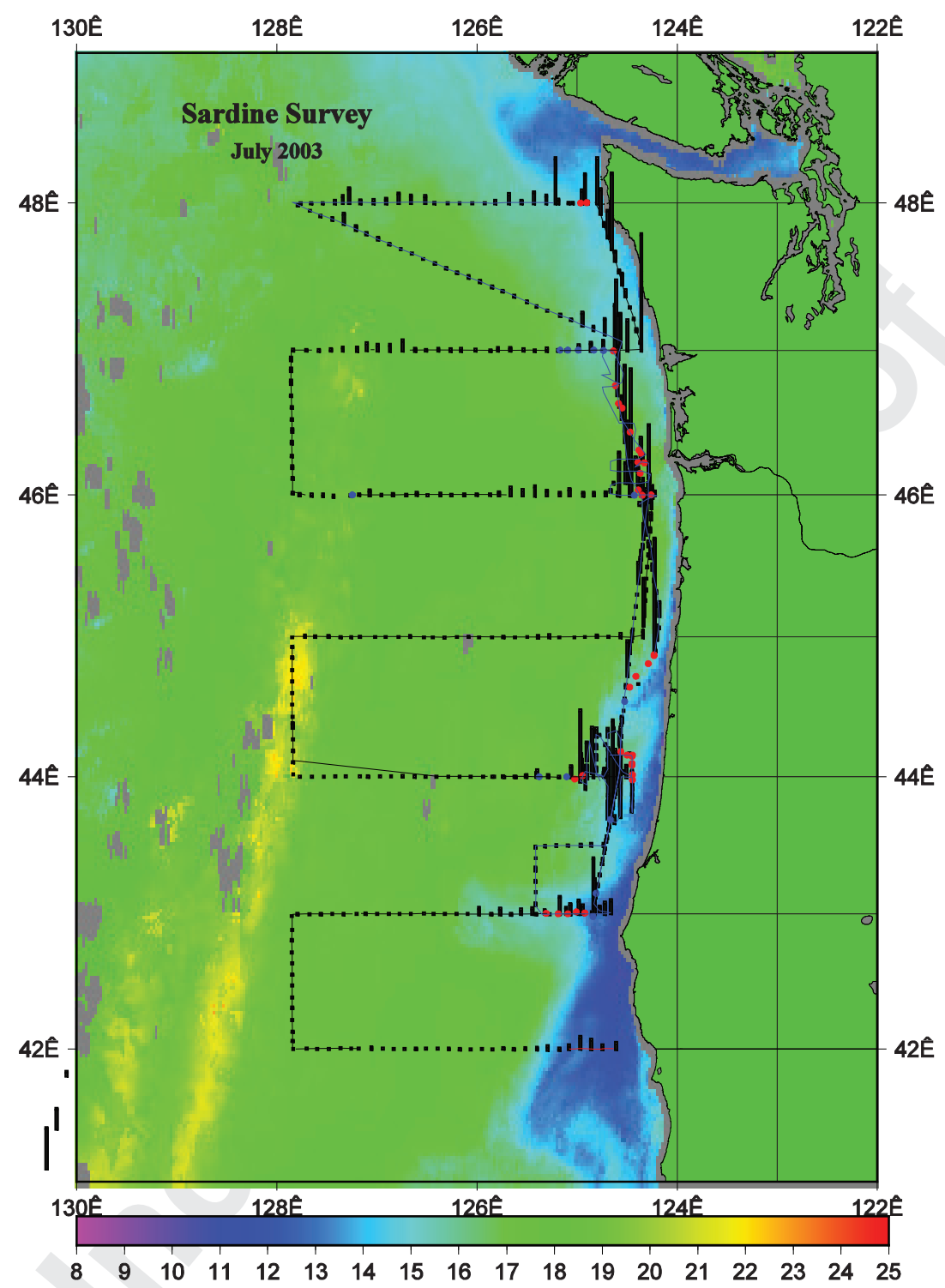

Fig. 19.5 Results of a sardine survey off the coast of Oregon and Washington in the northwestern United States. The background image presents satellite-derived sea-surface temperature $\left({ }^{\circ} \mathrm{C}\right)$ according to the color bar. Vertical bars along the survey tracks present relative acoustic return. Blue (red) circles mark locations of trawls that did (did not) contain sardines (Figure courtesy of Richard Charter, NOAA National Marine Fisheries Service) 
data collected from aircraft. To get a more complete picture of what is going on in an ecosystem, trawl data can be included to provide information on species, community dynamics, and size distributions. In addition, trawls provide a means to ground-truth visual observations on species identification from aircraft.

\subsection{The Future}

Advances in fisheries research will require data collected over a wide range of spatial and temporal scales and effective and efficient methods to meld large data volumes into a coherent picture of an ecosystem. Continuous monitoring of the earth's surface from space will continue with improved spatial resolution, more accurate measurements of primary production in coastal areas, and the addition of surfacesalinity measurements. Continuous monitoring of the water column at specific locations will only increase with the deployment of additional instrumented moorings. Episodic surveys using aircraft as sample platforms will increase and should improve the effectiveness of coordinated ship surveys. This increased use of aircraft as sample platforms will be driven by the synoptic nature of aerial surveys and their low cost per survey kilometer relative to ship surveys. The greatest challenge for researchers will be to effectively integrate and analyze these data sources.

The most straightforward way to combine techniques is to use adaptive surveys. Satellite and mooring data would be used to define the area and timing for the most effective aircraft and ship surveys. The area and timing of ship surveys are often based on historical information, but these may shift in the short term due to interannual variability in water temperatures and potentially become unreliable over longer time periods due to shifts in habitat associated with global climate change or local regime shifts. Aircraft surveys can be used to direct ship surveys to efficiently use ship time. To illustrate the latter, consider the case of Fig. 19.5. A lidar instrument package was flown over the ship during the systematic survey. Had the ship been directed by the lidar survey, it could have covered less than the full transects, but still captured most of the backscattered acoustic energy. Specifically, $90 \%$ of the acoustic energy could have been obtained from $65 \%$ of the survey track distance, or $65 \%$ of the energy at less than $30 \%$ of the survey distance. The result would be a significant reduction in survey cost with a minor decrease in accuracy. Alternatively, the same ship time could be directed to more productive areas, producing a more accurate survey at the same cost. Caveats to this strategy include the assumption that all species of interest are detected by both sensors, and that the time required by the ship to transit to high-density areas observed during the aerial survey are small relative to the time periods when animal distributions change significantly.

One difficult aspect when studying the interaction between distributions of marine animals and habitat components that affects distributions is obtaining data at high spatial and temporal scales. Historically, researchers studying nekton have relied on ship-based observations. It is now possible to integrate data from a variety 
of platforms. Increases in computing power and data storage capabilities allow researchers to manipulate and analyze large quantities of data. Initial data products include images or "snapshots" of an ecosystem. The Geographic Information System (GIS) is an important tool for fisheries biologists and is being used more frequently to display relationships between the distributions of pelagic marine life and important habitat components. Increased computing power also enables geostatistical tools to interpolate data values in areas where data were not collected (e.g., Reese and Brodeur 2006). Combining data from multiple platforms within a GIS framework facilitates a synoptic and complete coverage of important fish habitat. The production of images within GIS software can easily be shared with resource managers, scientists, politicians, and the general public.

Another way to combine techniques is to use statistical parameter estimation. For example, if we have statistical models of the probability density functions of lidar-returned energy, acoustic backscattered energy, and the trawl catches of sardine biomass, we can combine those data to predict the value of the sardine biomass. We want to predict sardine biomass $S$ such that the probability $p$ of biomass given observed lidar $L$, acoustic $A$, and trawl $T$ is a maximum (i.e., $p(S \mid L, A, T)]$. Using Bayes' theorem, this quantity will be maximized when $p(L, A, T \mid S) p(S)$ is at a maximum. The first factor describes the probability density of the observed signals for a given biomass and the second describes the probability density of the overall sardine biomass. The first factor includes all uncertainties within surveys such as under-sampling, fish detectability, and sensor noise. It is based on an understanding of the characteristics of the various techniques. The second factor is based on the variability in biomass from historical data.

Finally, different techniques might be combined using data assimilation into biophysical models (e.g., Besiktepe et al. 2003). Data can be inserted into the models at the temporal and spatial scales available. In a classical view of four-dimensional modeling, one specifies the conditions at all points at time zero and allows the model to develop from there to the time of interest, according to the appropriate differential equations. In a model that includes assimilation, the model predictions will be compared with observations made after the initial time of the model. Model results will be adjusted to more closely conform to the observations, and the model run will proceed. Consistency of the model (i.e., the requirement that it satisfy the underlying equations) can be used to ensure that different data streams are weighted according to their intrinsic reliability. For this approach to be effective, we need better models than those currently available (Methot 2007, this volume), and we will probably also need more computer power than has been available to date.

We have described a vision of the future that includes improvements in sampling, acoustic, and optical technologies. However, we think even greater advances are possible by combining the results from different techniques, each of which has different strengths and weaknesses. This will be particularly effective as results from different platforms are combined to provide a complete picture of an ecosystem over a greater range of spatial and temporal scales. The potential for this is most evident in the epipelagic ecosystem, which is accessible to observation from ships, moorings, autonomous vehicles, aircraft, and satellites. 


\section{[Au1] References}

Benoit-Bird KJ, Au WWL (2004) Diel migration dynamics of an island-associated soundscattering layer. Deep-Sea Res. 51:707-719

Besiktepe ST, Lermusiaux PFJ, Robinson AR (2003) Coupled physical and biogeochemical data-driven simulations of Massachusetts Bay in late summer: real-time and postcruise data assimilation. J Mar Syst 40-41:171-212

Brown E D, Churnside JH, Collins RL, et al. (2002) Remote sensing of capelin and other biological features in the North Pacific using lidar and video technology. ICES J Mar Sci 59: $1120-1130$

Carrera P, Churnside JH, Boyra G, et al. (2006) Comparison of airborne lidar with echosounders: a case study in the coastal Atlantic waters of southern Europe. ICES J Mar Sci 63:1736-1750

Churnside JH, Demer DA, Mahmoudi B (2003) A comparison of lidar and echosounder measurements of fish schools in the Gulf of Mexico. ICES J Mar Sci 60:147-154

Churnside JH, Sawada K, Okumura T (2001) A comparison of airborne lidar and echo sounder performance in fisheries. J Mar Acoust Soc Jpn 28:49-61

Churnside JH, Thorne RE (2005) Comparison of airborne lidar measurements with $420 \mathrm{kHz}$ echosounder measurements of zooplankton. Appl Opt 44:5504-5511

Churnside JH, Wilson JJ (2004) Airborne lidar imaging of salmon. Appl Opt 43:1416-1424

Churnside JH, Wilson JJ, Tatarskii VV (2001) Airborne lidar for fisheries applications. Opt Eng 40:406-414

Fréon P, Misund OA (1999) Dynamics of pelagic fish distribution and behaviour: effects on fisheries and stock assessments. Fishing News Books, Oxford

Gunderson DR (1993) Surveys of fisheries resources. Wiley, New York

Lo NCH, Hunter JR, Churnside JH (2000) Modeling statistical performance of an airborne lidar survey for anchovy. Fish Bull 98:264-282

Onsrud MSR, Kaartvedt S, Breien MT (2005) In situ swimming speed and swimming behaviour of fish feeding on the krill Meganyctiphanes norvegica. Can J Fish Aquat Sci 62:1822-1832

Reese DC, Brodeur RD (2006) Identifying and characterizing biological hotspots in the Northern California Current. Deep-Sea Res II 53:291-314

Skaret G, Slotte A, Handegard NO, et al. (2006) Pre-spawning herring in a protected area showed only moderate reaction to a surveying vessel. Fish Res 78:359-367

Tenningen E, Churnside JH, Slotte A, et al. (2006) Lidar target-strength measurements on northeast Atlantic mackerel (Scomber scombrus). ICES J Mar Sci 63:677-682

Vasilkov AP, Goldin YA, Gureev BA, et al. (2001) Airborne polarized lidar detection of scattering layers in the ocean. Appl Opt 40:4353-4364

Wiebe PH, Stanton TK, Greene CH, et al. (2002) BIOMAPER-II: an integrated instrument platform for coupled biological and physical measurements in coastal and oceanic regimes. IEEE J Ocean Eng 27(3):700-716

Zorn HM, Churnside JH, Oliver CW (2000) Laser safety thresholds for cetaceans and pinnipeds. Mar Mammal Sci 16:186-200

\section{Author Query}

[Au1]: References indicating "complete" have been removed from the list and the relevant information added in the text 